\title{
Detection of object onsets and offsets: Does the primacy of onset persist even with bias for detecting offset?
}

\author{
Maria J. Donaldson $^{1}$ - Naohide Yamamoto ${ }^{1,2}$
}

Published online: 5 August 2016

(C) The Psychonomic Society, Inc. 2016

\begin{abstract}
Onset primacy is a robust visual phenomenon in which appearance of new objects (onsets) in a scene more effectively captures observers' attention compared with disappearance of previously viewed objects (offsets). We hypothesized that the human attentional system is programmed by default to prioritize the processing of onsets, because quick detection of them is advantageous in most situations. However, the attentional priority may be able to flexibly adapt to the detection of object offsets depending on observers' behavioral goals. To test these hypotheses, two experiments were conducted in which participants were biased toward finding offset of an existing object through top-down and bottom-up manipulations. Results showed that although onset primacy was reduced to some degree under strong offset bias, in general participants continued to detect onsets efficiently. These findings did not eliminate the possibility of attentional flexibility, but they do demonstrate the robustness of onset primacy, suggesting that environmental demands or motivational factors would need to be sufficiently strong for people to switch to an adaptive attentional mode.
\end{abstract}

Keywords Onset primacy $\cdot$ Cognitive control $\cdot$ Attentional modulation $\cdot$ Change detection

Maria J. Donaldson

m.j.donaldson@vikes.csuohio.edu

1 Department of Psychology, Cleveland State University, 2121 Euclid Avenue, Cleveland, $\mathrm{OH} 44115$, USA

2 School of Psychology and Counselling and Institute of Health and Biomedical Innovation, Queensland University of Technology, Brisbane City, Australia
The visual world is a dynamic, ever-changing environment. To promote successful interaction with the environment, the human attentional system prioritizes the processing of certain visual events over others. When a visual event occurs in the absence of attentional focus, its occurrence often is missed or detection of its presence is delayed. This phenomenon has been coined change blindness (Simons \& Rensink, 2005). For example, movie viewers may miss the presence of bloopers and other mistakes the editors may have overlooked. Additionally, a distracted driver may fail to notice another vehicle on a road. Failure to notice such visual changes can lead to grave consequences. Although it may appear that we are vigilant to our surroundings, attend to details, and notice the people around us, attention is not an unlimited resource, and there are aspects of the environment that we may miss.

To evaluate how the prioritization of visual events unfolds, it is helpful to consider research using visual search paradigms that focus on attentional capture. One type of task used in the visual search domain requires participants to identify an abrupt change in the search display, wherein the target stimulus is either the appearance of a new object (object onset) or the disappearance of a previously viewed object (object offset). Both types of visual changes evoke similar sensory changes, including potential changes in luminance, as well as visual transients in the display associated with the change. Despite these sensory similarities, object onsets and offsets are not attended to equally when processing a visual array. Results from studies assessing visual search and attentional capture have consistently revealed that onsets are detected with greater speed and accuracy than object offsets (Chua, 2013; Yantis \& Jonides, 1984). This phenomenon has been described as onset primacy in the literature (Cole, Kentridge, \& Heywood, 2004).

Although onset primacy was first noted in visual search, support for onset primacy has been found in other visual 
paradigms such as change blindness. It is important to consider onset primacy within contexts other than visual search because change blindness tasks are qualitatively different than most visual search tasks. In a typical visual search study, participants are instructed to look for a pre-specified target stimulus among other distractor symbols, shapes, or letters within a visual array. Generally, the array is continuously presented until the target is located; thus, this task assesses attentional functioning without strong demands on memory ability (Yantis \& Jonides, 1984). Rarely does attentional processing occur in the absence of memory; however, so extending the onset primacy phenomenon to other areas of visual attention, such as change blindness, provides richer contexts under which multiple cognitive processes may interact to simulate how attentional capture in the real world is most likely to occur.

Early studies on change blindness mainly involved instances of unexpected object replacement (Levin \& Varakin, 2004), but change blindness research has more recently focused on object onsets and offsets, because they naturally occur in the environment compared with other types of visual change events (Cole et al., 2004; Cole, Liversedge, \& Simon 2006). Notably, Cole, Kentridge, Gellatly, and Heywood (2003) used a one-shot flicker paradigm in which two images are presented in succession, separated by a gray screen, for only one cycle. In each trial, from the first image to the second image, either an object onset or offset occurred, and observers made a button press to indicate the location of the change. In one task, the stimuli used were computer-generated two-dimensional shapes of different colors and sizes presented against a uniform background. In another task, real-world objects, such as paper clips and floppy disks, were used instead of simple color patches, but stimuli were still two-dimensional aerial views of the arrangements of these objects. Cole et al. (2003) found that observers performed this task more quickly and accurately when they experienced object onsets than offsets, demonstrating onset primacy in the form of greater resistance to change blindness. Donaldson and Yamamoto (2012) replicated this finding by using three-dimensional scenes with naturally occurring visual cues such as depth cues and texture gradient, illustrating the robustness of onset primacy in change blindness. These studies suggest that the prioritization of relevant visual events, and specifically onset primacy, is a robust occurrence applicable to many domains of visual attention.

Cole et al. (2003) and Donaldson and Yamamoto (2012) clearly demonstrated that onset primacy takes place in a variety of viewing conditions, but the question remains as to why onsets are superior to offsets in effectively capturing visual attention. The underlying mechanisms of onset primacy are actively debated and perhaps not fully understood. Evidence from some studies within the visual search domain indicates that the object onset itself, rather than other concomitant visual changes, ultimately captures an observer's attention (Cole et al., 2006; Davoli, Suszko, \& Abrams, 2007; Jonides \& Yantis, 1988; Yantis \& Jonides, 1984; see also Franconeri, Hollingworth, \& Simons, 2005; Hollingworth, Simons, \& Franconeri, 2010). In other words, from this perspective, there is something special or unique about object onsets above and beyond sensory-level changes, because these changes also cooccur with object offsets. According to Cole et al. (2003), this uniqueness of object onsets might rest upon the fact that rapid detection of abrupt onsets promotes survival. Until an onset captures attention, it is unknown if its quality is benign or threatening, and organisms must be able to react to threat efficiently. For this reason, it is beneficial for an animal to locate and avoid a predator entering its surroundings, rather than paying attention to when the predator leaves. Similarly, it is more important for automobile drivers to notice another car suddenly entering their field of view than a pedestrian walking away. Thus, from an evolutionary perspective, there may be adaptive reasons for onset to be the primary type of visual events to which attention is directed. This conjecture provides a theoretical basis for the idea that in the absence of specific behavioral goals or motivational factors, the attentional system defaults to prioritization of onset detection (Folk, Remington, \& Johnston, 1992). Taken together, we formulate the default mode hypothesis, which posits that the natural selection shaped the human attentional system for the ecological utility in paying increased attention to object onsets.

It should be noted, however, that everyday life also includes scenarios in which object offset has heightened importance. For example, in cases of lifeguarding or parenting, it may be more important to notice a missing child, as opposed to the child coming back in sight. Similarly, it is advantageous for a sales clerk to identify missing merchandise. In these situations, observers may be able to allocate their attentional resources to detection of offset (or, more generally, non-onset) events. This is the attentional modulation hypothesis, which states that observers give attentional priority to a particular type of visual events (whether it is onset, offset, or anything else) that is most relevant to the current environment of the observers.

According to the default mode hypothesis, onset primacy is an intrinsic property of the human attentional system. It is set at directing increased attention to object onsets because detecting the appearance of new objects quickly and accurately is generally the most effective way of interacting with an environment. Therefore, even when detection of other types of visual events, such as object offsets, can be of higher priority, observers might continue allocating attention to object onsets (Cole \& Kuhn, 2010). By contrast, the attentional modulation hypothesis postulates that observers flexibly adjust their attentional system to better detect non-onset events when doing so is more appropriate to the observers' goals. The default mode hypothesis, in its strictest form, might not allow for any 
flexible prioritization of non-onset events. Yet, substantial research (Folk et al., 1992; Yantis \& Jonides, 1990) indicates that humans are capable of resisting attentional capture by onsets if attention is sufficiently focused on another task or goal, providing an empirical basis upon which the attentional modulation hypothesis stands.

The purpose of the present study was to test the predictions of the default mode and attentional modulation hypotheses by creating a situation in which observers were biased toward detecting object offsets. This study examined these two hypotheses by applying them to change blindness in realistic scenes, extending the scope of the hypotheses that largely drew upon findings from visual search studies (Cole \& Kuhn, 2010; Folk et al., 1992; Yantis \& Jonides, 1984, 1990). Donaldson and Yamamoto (2012) found that with no attentional manipulation, onset primacy is observable in a change blindness paradigm using images with threedimensional visual cues that simulate a real-world environment. With this earlier study as a backdrop, the present study sought to determine how human attention mechanisms may enable or prohibit flexible attentional prioritization when detecting object onsets and offsets in realistic scenes. In two experiments, the bias in favor of object offset was induced in participants to assess whether object offsets can be detected more efficiently compared to when participants have no such bias (in that case, they should use the default mode of attention with which they naturally look for object onsets). If participants' ability to detect offsets was improved by the offset bias, it would support the attentional modulation hypothesis. This improvement might come at the cost of impaired onset detection due to a shift from the default attentional mode. On the other hand, if onsets continued to be detected with higher accuracy and shorter reaction time despite exposure to the offset bias, this pattern of results would support the default mode hypothesis.

\section{Experiment 1}

\section{Method}

Participants Ninety participants (21 men, 69 women) from the Cleveland State University community consented to participate in this study for partial course credit. They ranged in age from 18 to 47 years $(M=20.47)$. All participants reported normal or corrected-to-normal vision.

Materials Experimental stimuli presented to the participants were color digital pictures that depicted a wooden round tabletop on which eight objects were placed in various arrangements. The objects were toys and small household items that were approximately $4 \mathrm{~cm}$ in width, $2 \mathrm{~cm}$ in depth, and $3 \mathrm{~cm}$ in height. The tabletop was $38 \mathrm{~cm}$ in diameter and supported by a table base that was $75-\mathrm{cm}$ tall. The objects were placed so that half were on the left side of the tabletop and half were on the right side. No object occluded another object, such that every object was visible in its entirety. The walls behind and to either side of the table were visible, as was the carpet on which the table stood. Digital pictures of the table were taken from an angle of approximately $30^{\circ}$, which provided a naturalistic view of the object arrangements. For examples of the stimuli, see Fig. 1.

These images were presented on a 17-inch liquid crystal display. The screen was positioned vertically in front of the participant. The distance between the participant and the screen

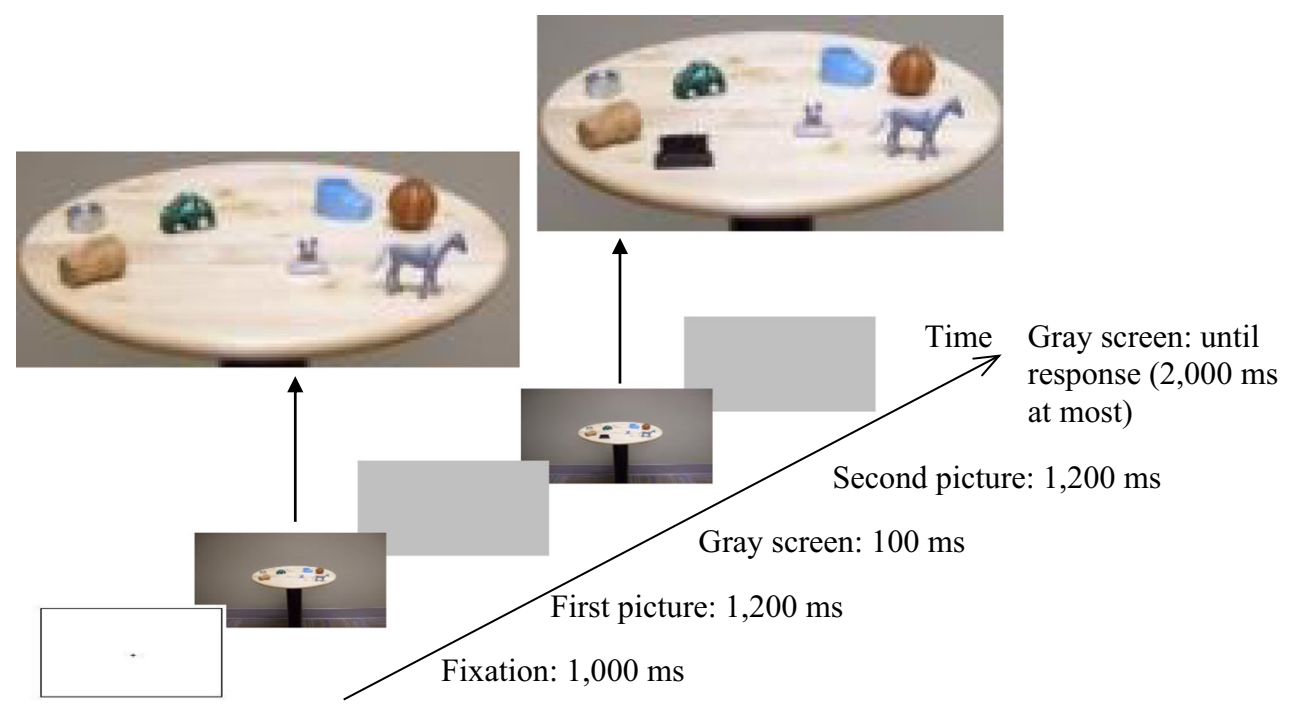

Fig. 1 Trial sequence. In this example, an object (the brush) is added to the second scene on the left-hand side. This is an onset trial. Pictures in the trial sequence are actual pictures that were used in the experiments. Larger pictures are close-up views of the object array 
was approximately $60 \mathrm{~cm}$. The images occupied the entire screen and subtended approximately $35^{\circ} \times 40^{\circ}$ of visual angle. The tabletop subtended approximately $10^{\circ} \times 18^{\circ}$, and on average, the objects subtended $1.7^{\circ} \times 2.3^{\circ}$. When presented on the screen, the center of the tabletop was approximately at the center of the screen so that the left and right halves of the tabletop corresponded to those of the screen.

Design Participants were randomly assigned to one of four conditions that will be described in detail later. The only constraint to random assignment was that each condition had 20 participants (as noted below, data from 10 participants were excluded from the analysis, resulting in having 80 participants in total). All conditions began with a short practice session of 16 trials. Stimuli used in the practice session were in a unique configuration that was not repeated in subsequent blocks. Photographs used in the experimental blocks depicted the same objects, but different configurations were used. These manipulations ensured that participants were not influenced by any adventitious priming effects that can result from seeing particular objects or configurations more frequently (Chun \& Jiang, 1998).

Following the practice session, all participants went through two blocks of trials (referred to as Blocks 1 and 2 hereafter). In each block, participants viewed a series of 160 photograph pairs. Two different sets of 160 photograph pairs were used in Blocks 1 and 2. Two photographs of a pair depicted the same object configuration, but one change occurred between them-a new object appeared in the second photograph (onset), or one of the objects in the first photograph disappeared in the second photograph (offset). Of the 160 pairs, 128 pairs created experimental trials in which the same eight objects were used. Unless otherwise noted below, one onset trial and one offset trial were created from the same two photographs by reversing the order of their presentationthat is, in an onset trial, a seven-object image was shown first, followed by an eight-object image; and in an offset trial, the eight-object image preceded the seven-object image. This manipulation ensured that the identical visual characteristics were present in onset and offset trials. The remaining 32 pairs were used as dummy trials. In a dummy onset trial, a ninth object was added to the second photograph in the pair, and in a dummy offset trial, one of seven objects in the first photograph was removed in the second photograph. This manipulation was to prevent participants from anticipating the trial type based on the number of objects present in the first photograph of the pair. The onset and offset trials (both dummy and experimental trials) were randomly intermixed, unless otherwise noted below. Each object was used the same number of times to create an onset trial or an offset trial throughout the experiment (i.e., all objects were presented an equal number of times throughout the experiment).
The participants' task was to detect the change as accurately and quickly as possible by indicating whether it occurred in the right half or the left half of the tabletop. The location of the change was counterbalanced such that in half the onset trials objects in the left side changed and in the remaining half the objects in the right side changed. The same was done for offset trials.

The conditions differed as to whether and how participants experienced bias toward detecting object offsets. The bias was induced either during the first block of trials (Block 1) or before the practice session (details are described below). As such, not all conditions used the same practice session or Block 1. On the other hand, the four conditions were identical in the second block (Block 2), which was designed as described above.

Neutral condition In this condition, no particular bias was induced in participants. In other words, it was expected that they would exhibit onset primacy as a result of using the default attentional mode. The practice session included eight onset trials and eight offset trials. The order of these trials was randomly determined. In Block 1, there were 64 onset trials and 64 offset trials that were also randomly intermixed. There were 16 dummy onset trials and 16 dummy offset trials randomly distributed throughout the block.

Skewed ratio condition In this condition, the offset bias was induced by showing participants more offset trials than onset trials in Block 1. After receiving 16 practice trials (12 offset and 4 onset), participants viewed 100 offset trials and 28 onset trials in Block 1. Furthermore, the first 20 trials and the last 20 trials in this block were offset trials while the remaining 88 trials presented both onsets and offsets (randomly intermixed). In Block 1, 24 of the dummy trials were offsets, and only 8 were onsets. Seventy-two photograph pairs were used only as offset trials. Twenty-eight photograph pairs were used for creating both offset and onset trials. The objects and side of the screen on which the change occurred were still counterbalanced. These manipulations were intended to have participants perceive object offset as the primary type of change that they would encounter in the experiment so that they would allocate more attention to offsets than to onsets. It has been shown that manipulation of the ratio of different trial types is generally effective for leading participants to adopt a particular strategy depending on which type of trials is dominant (Bugg, Jacoby, \& Toth, 2008).

Skewed ratio with instruction condition This condition was identical to the skewed ratio condition with an exception that participants were given an additional instruction to pay closer attention to offset trials than onset trials. Participants read the instruction on the computer screen, which explicitly stated that people generally find appearances of new objects more 
quickly and accurately than disappearances of previously viewed objects, but that in this experiment, participants should aim to be faster and more accurate in offset trials because they are more important in this context, while still responding to onset trials. The instruction was added to determine if this topdown expectation would assist in creating a stronger bias toward offsets.

Instruction only condition In this condition, participants were given the same additional instruction used in the skewed ratio with instruction condition. However, aside from this instruction, the design of this condition was identical to that of the neutral condition.

Procedure This experiment used the same procedure as in Donaldson and Yamamoto (2012), which adopted the oneshot flicker paradigm developed by Cole et al. (2003). Participants sat in front of a computer screen, centered in front of a keyboard. The participants were told that they would be viewing a series of photograph pairs in which an object would change between two images of each pair. They also were instructed that the change would be either an onset of a new object or an offset of an existing object. They were instructed to press either the "F" key if the change occurred on the left side of the screen or the "J" key if the change occurred on the right side of the screen. They used their left index finger to press the left button and their right index finger to press the right button. They were cautioned to be as quick, but also as accurate as possible. They were run individually.

Figure 1 illustrates the trial sequence. In each trial, participants first viewed a fixation cross for $1,000 \mathrm{~ms}$ that was presented at the center of the screen. They were instructed to keep fixating on the cross while it was displayed and maintain their fixation around the same area after the cross disappeared. They then viewed a first image for 1,200 ms. This image was followed by a 100 -ms gray screen that produced the one-shot flicker of the scene. The second image was then displayed for $1,200 \mathrm{~ms}$. At the onset of the second image, participants were allowed to make a button press indicating which side of the screen the change occurred. Following the presentation of the second image, the second gray screen was displayed until the participant made his or her response or until 2,000 ms had passed. Reaction time was recorded between the appearance of the second image and the participant's button press. Accuracy in the left/right judgment also was measured based on participants' button press response. When the participant made an error in the left/right judgment, reaction time from such a trial was not included in reaction time analyses.

Data analysis Data were analyzed by a 2 (trial type: onset and offset) $\times 4$ (attentional bias: neutral, skewed ratio, skewed ratio with instruction, and instruction only) mixed analysis of variance (ANOVA), which was separately conducted for reaction time and accuracy, for both Block 1 and Block 2 . Trial type was a within-participant factor, and attentional bias was a between-participant factor. Unless noted otherwise, the sphericity assumption was met in ANOVAs reported in this article.

\section{Results}

Participants were excluded from the analysis if their mean accuracy in the left/right judgment task for either onset or offset trials was more than three standard deviations away from the mean of all participants for a given attentional bias condition. Six participants were excluded on the basis of accuracy data (two from the neutral condition, two from the skewed ratio condition, one from the skewed ratio with instruction condition, and one from the instruction only condition). Their mean accuracy for onset and offset trials were $77.09 \%$ and $71.43 \%$, respectively. The pattern of results obtained in this experiment did not vary significantly when these individuals were included in analyses. In addition, data from three participants in the skewed ratio condition were not analyzed because miscommunication of instructions or simple fatigue prevented task completion. Reaction time outliers were then removed if they were more than three standard deviations away from each participant's mean, separately for onsets and offsets. There were no outliers at the lower cutoff. One to three outliers exceeding the upper cutoff were removed for 57 participants. They were removed to eliminate errors of anticipation and inattention, but exclusion of the outliers did not alter the results. Removal of these outliers did not count against participants' accuracy data. Mean reaction times were then computed for each trial type and for each attentional bias condition, based on trials in which correct responses were made.

Block 1 Data from the first block were analyzed to determine if experimental manipulations were effective in inducing offset bias in participants. Although these data did not allow strict comparisons between attentional bias conditions because there were differing amounts of onset and offset trials across the conditions, this analysis still provided information about the strength of the biasing manipulations.

Reaction time Figure 2a shows mean reaction times in Block 1 as a function of trial type and attentional bias condition. As shown in the figure, participants detected onsets more quickly than offsets in the neutral condition, but in the other three conditions (i.e., with some offset-detection bias), mean reaction times to offset trials were numerically shorter than those to onset trials. In the ANOVA, there was neither the main effect of attentional bias, $F(3,76)=0.232, p=0.874, \eta_{\mathrm{p}}{ }^{2}=$ 0.009 , nor the main effect of trial type, $F(1,76)=0.414, p=$ 

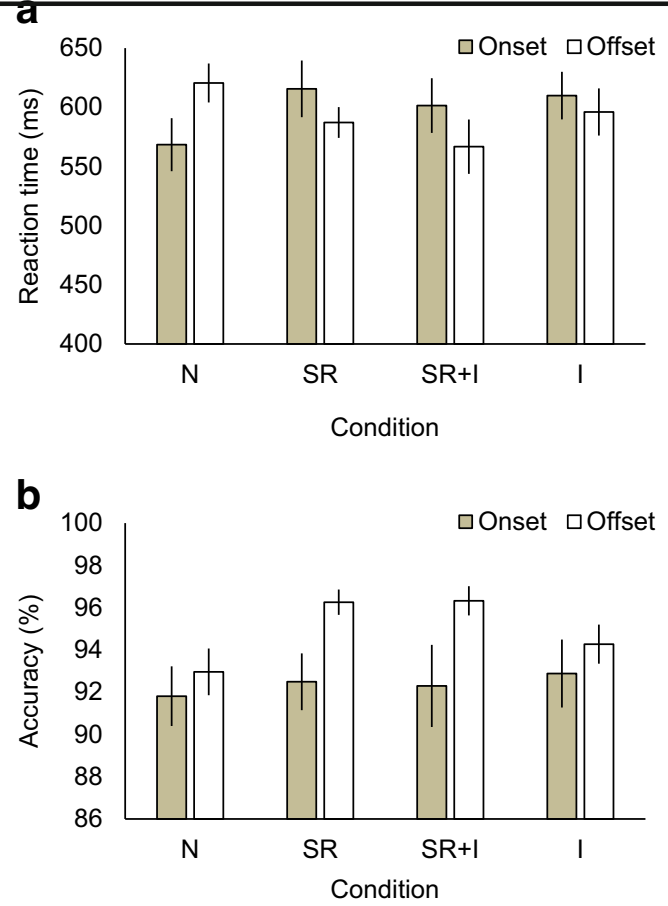

Fig. 2 Mean reaction time (a) and accuracy (b) in the first block of trials in Experiment 1 as a function of attentional bias condition and trial type. Condition labels are defined as follows: $\mathrm{N}=$ neutral condition; $\mathrm{SR}=$ skewed ratio condition; $\mathrm{SR}+\mathrm{I}=$ skewed ratio with instruction condition; and $\mathrm{I}=$ instruction only condition. Error bars represent \pm 1 standard error of the mean

$0.522, \eta_{\mathrm{p}}{ }^{2}=0.005$, indicating that in the first block there was no overall tendency to detect one type of trials faster than the other. The interaction of trial type and attentional bias was significant, however, $F(3,76)=5.271, p=0.002, \eta_{\mathrm{p}}{ }^{2}=$ 0.172 . Simple effects analyses revealed significantly shorter reaction times to onset trials than to offset trials in the neutral condition, $p=0.004$, and significantly shorter reaction times to offset trials than to onset trials in the skewed ratio with instruction condition, $p=0.048$. (All $p$-values from simple effects analyses in this study are corrected for multiple comparisons by the Bonferroni procedure and can be evaluated against $\alpha=0.05$.) These analyses also showed that reaction times to onset and offset trials in the other two conditions were statistically equivalent (skewed ratio: $p=0.104$; instruction only: $p=0.518$ ). These results suggest that biasing manipulations did exert observable effects - reliable onset primacy was present in the neutral condition, but it was eliminated by greater exposure to offset trials (the skewed ratio condition) and instruction to focus on offset trials (the instruction only condition); and when these two were combined (the skewed ratio with instruction condition), they were able to promote clear offset bias.

Accuracy Figure $2 \mathrm{~b}$ shows mean accuracy in Block 1 as a function of trial type and attentional bias condition. Generally, onset and offset trials did not differ greatly, as consistent with previous results that accuracy in this paradigm was not as sensitive as reaction times to the effects of object onsets and offsets (Donaldson \& Yamamoto, 2012). In the ANOVA, the main effect of trial type was significant, $F(1,76)=14.286, p<$ $0.001, \eta_{\mathrm{p}}{ }^{2}=0.158$, such that there was higher accuracy on offset trials compared with onset trials. However, neither the main effect of attentional bias nor the interaction between attentional bias and trial type were significant, $F(3,76)=$ $0.762, p=0.519, \eta_{\mathrm{p}}{ }^{2}=0.029$, and $F(3,76)=1.066, p=$ $0.369, \eta_{\mathrm{p}}{ }^{2}=0.040$, respectively. Although the four attentional bias conditions were deemed statistically indistinguishable, the difference between onset and offset trials was more pronounced for the conditions in which offset bias was induced. This pattern of the accuracy data is consistent with the interpretation that the manipulations were effective enough for modulating onset primacy.

Block 2 Data from the second block were analyzed to examine the effect of the offset-biasing manipulations on onset and offset detection. All attentional bias conditions used identical Block 2 in which there were equal amounts of onset and offset trials, enabling a strict comparison of results between the conditions.

Reaction time Mean reaction times are plotted in Fig. 3a as a function of trial type and attentional bias condition. Although participants in the skewed ratio, skewed ratio with instruction, and instruction only conditions were biased toward directing greater attention to offset detection, onset trials still yielded shorter reaction times in all of these conditions. Consistent with this observation, neither the interaction between trial type and attentional bias nor the main effect of attentional bias were significant, $F(3,76)=0.526, p=0.666, \eta_{\mathrm{p}}{ }^{2}=0.020$, and $F(3$, $76)=0.296, p=0.828, \eta_{\mathrm{p}}{ }^{2}=0.012$, respectively. The main effect of trial type was significant and had a large effect size, $F(1,76)=114.908, p<0.001, \eta_{\mathrm{p}}{ }^{2}=0.602$, showing that robust onset primacy was present in all four conditions.

Accuracy Mean accuracy scores are plotted in Fig. 3b as a function of trial type and attentional bias condition. In spite of the offset bias manipulation, all conditions yielded similar accuracy data - although onset trials yielded higher accuracy than offset trials, neither the interaction between trial type and attentional bias nor the main effect of attentional bias were significant, $F(3,76)=0.212, p=0.888, \eta_{\mathrm{p}}{ }^{2}=0.008$, and $F(3,76)=1.682, p=.178, \eta_{\mathrm{p}}{ }^{2}=0.062$, respectively. The main effect of trial type also was not significant, $F(1,76)=1.951, p$ $=0.167, \eta_{\mathrm{p}}{ }^{2}=0.025$. These data, like accuracy data in Block 1 , showed that accuracy was less sensitive than reaction times in the current paradigm (Donaldson \& Yamamoto, 2012). Importantly, however, the fact that onset trials generally yielded greater accuracy excludes the possibility that speedaccuracy tradeoffs affected patterns of the reaction time data discussed above. 

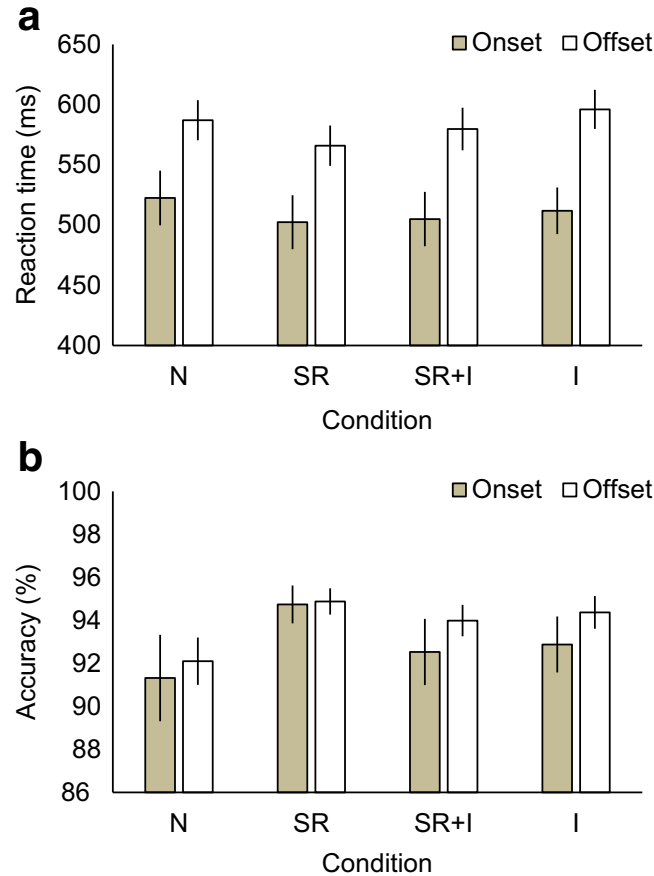

Fig. 3 Mean reaction time (a) and accuracy (b) in the second block of trials in Experiment 1 as a function of attentional bias condition and trial type. Condition labels are defined as follows: $\mathrm{N}=$ neutral condition; $\mathrm{SR}=$ skewed ratio condition; $\mathrm{SR}+\mathrm{I}=$ skewed ratio with instruction condition; and $\mathrm{I}=$ instruction only condition. Error bars represent \pm 1 standard error of the mean
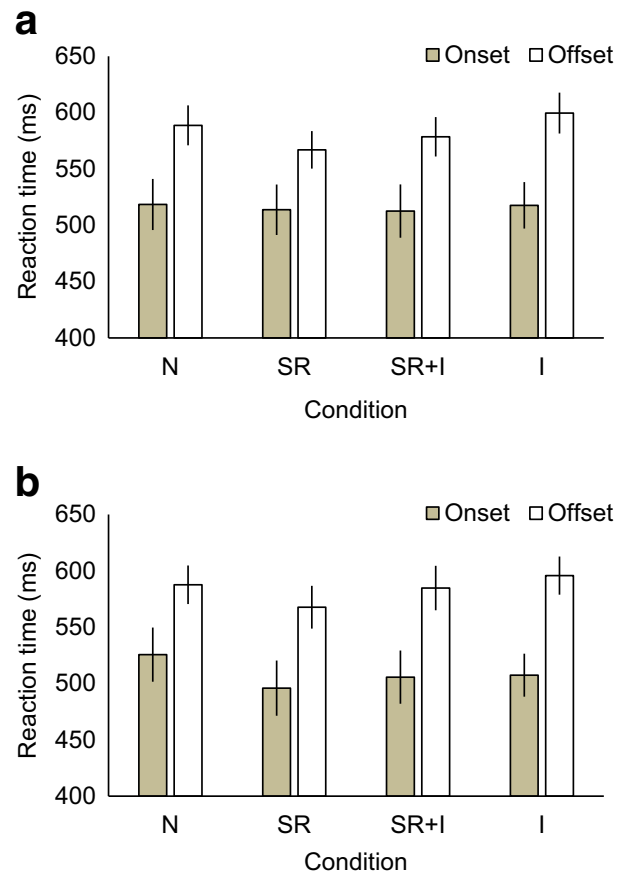

Fig. 4 Mean reaction time (a and b) and accuracy (c and $\mathbf{d}$ ) in the first and second halves of the second block of trials of Experiment 1 as a function of attentional bias condition and trial type. Panels $\mathrm{A}$ and $\mathrm{C}$ show data from the first half, and panels $\mathrm{B}$ and $\mathrm{D}$ show data from the
Split-block analysis As shown above, Block 2 results generally indicated that there was no effect of offset bias manipulations. Because there was evidence that the manipulations produced observable effects in Block 1, one possibility is that the offset-prioritizing effects were still present in early trials of Block 2 and then dissipated later in the block. To test this idea, a split-half analysis was conducted on Block 2 data. For this analysis, performance on the first 80 and last 80 trials within the block was compared. Because of the random presentation of onset and offset trials (and experimental and dummy trials), the numbers of onset and offset trials used for this analysis were not identical between participants as well as between the first half and the second half of the block. However, it was confirmed that onset and offset trials were evenly distributed across participants and the two halves of the block. The mean numbers of onset and offset trials in each half of the block were as follows: 31.46 onset and 32.74 offset trials in the first half; and 32.54 onset and 31.25 offset trials in the second half.

Figure 4 displays mean reaction times and accuracy in each half of Block 2 as a function of trial type and attentional bias condition. Overall, the patterns of data (both reaction time and accuracy) in the two halves closely resembled each other, with the possible exception of accuracy in the skewed ratio condition. To examine whether this observation can be statistically qualified, two mixed ANOVAs were run (one for reaction time and one for accuracy) with trial type and sub-block (first half and second half) as within-participant factors and

\section{C}

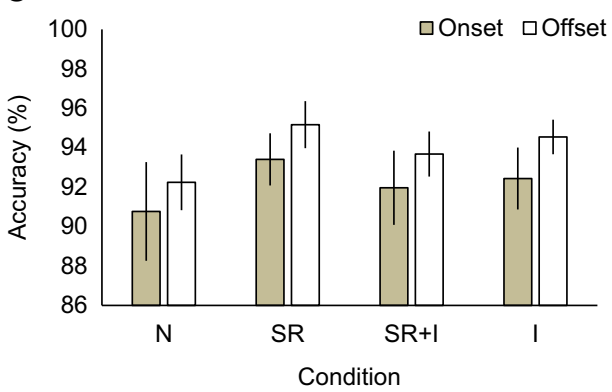

d

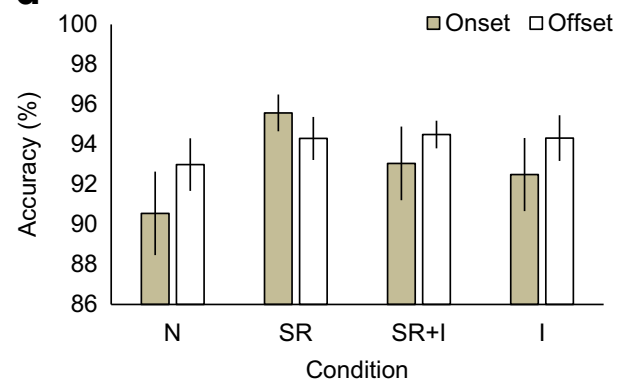

second half. Condition labels are defined as follows: $\mathrm{N}=$ neutral condition; $\mathrm{SR}=$ skewed ratio condition; $\mathrm{SR}+\mathrm{I}=$ skewed ratio with instruction condition; and I = instruction only condition. Error bars represent \pm 1 standard error of the mean 
attentional bias as a between-participant factor. In these ANOVAs, all main effects and interactions including the sub-block variable failed to reach significance for both reaction time and accuracy, $F \mathrm{~s}(1,76)<1.312, p \mathrm{~s}>0.255, \eta_{\mathrm{p}}{ }^{2} \mathrm{~s}<$ 0.031 , suggesting that results did not differ between early and late trials. In these analyses, only the main effects of trial type were significant, $F(1,76)=119.684, p<0.001, \eta_{\mathrm{p}}{ }^{2}=0.612$ (reaction time) and $F(1,76)=4.997, p=0.028, \eta_{\mathrm{p}}{ }^{2}=0.082$ (accuracy). Thus, it was concluded that Block 2 data provided no evidence for prioritization of offset detection, even in the early trials.

\section{Discussion}

Results from Experiment 1 showed that experimental manipulations did produce offset-detection bias (in the form of diminished onset primacy) while the manipulations were being implemented in the first block. However, participants still detected onsets more quickly than offsets in the immediately following second block. These results may partially support both the attentional modulation hypothesis and the default mode hypothesis by showing that across a relatively small number of trials over a short amount of time, the attentional system adapted to environments prioritizing non-onset events, and also by demonstrating that the increased attention to nononset events disappeared quickly and participants immediately reverted back to the default onset-prioritizing mode in Block 2. Perhaps this shift-back to onset primacy in the skewed ratio and skewed ratio with instruction conditions stemmed from the equivalent ratio of onset and offset trials in Block 2. For participants in these conditions, ongoing dominance of offset trials might have been necessary to maintain prioritization of offset detection. Similarly, temporal continuity between blocks might have been needed for the effect of instruction to be carried over into Block 2 (there was a brief disruption between the blocks). In any case, the present results lead to the conclusion that attention to onsets and offsets can be modulated but only transiently, and it does not vary in any more persistent manner as a function of environmental demands or motivational factors that call for better detection of non-onset events.

Before reaching this conclusion, however, two alternative explanations for the current results must be given careful consideration. For one thing, it is possible that the quick reversion to onset primacy was observed because Block 1 did not provide sufficiently strong training for prioritizing offset detection. In fact, when Leber and Egeth (2006a) implemented a similar paradigm in a visual search study in which participants' attention was biased toward certain features of search targets, a training block of 320 trials was sufficient to make the bias persist in a subsequent test block of 320 trials. On the other hand, when the training block was shortened to 40 trials, there was no carryover of the bias into the test block. In addition, more generally, when participants are trained to pay attention to particular kinds of information through practice, it often is the case that several hundreds of trials are needed before the effect of practice becomes evident (Kelley \& Yantis, 2009). Given that Experiment 1 used a relatively short training block (i.e., Block 1 containing 160 trials), the possibility remains that with more extensive training, the effects of offset-biasing manipulations may be reliably maintained in Block 2. To address this issue, Experiment 2 was conducted using 320 trials in each block of the experiment while replicating other aspects of the design and procedure of Experiment 1.

The other alternative explanation is that the present experiment might have lacked sufficient statistical power to detect the possible effects of offset-biasing manipulations in Block 2. The main (but tentative) conclusion of the experiment, which states that onset primacy was observed equally in all conditions in Block 2, hinges on the absence of significant interaction between trial type and attentional bias condition in Block 2. Thus, for this conclusion to be held up, it is critical that the experiment was powerful enough to identify this interaction, had it actually been present. Experiment 2 was performed to address this issue as well by conducting a priori power analysis and ensuring adequate power of the experiment. Results from Experiment 1 provided a solid empirical basis for estimating the effect size of the interaction, establishing the validity of this power analysis.

It may be worth noting that the two alternative explanations discussed above are not mutually exclusive; it is possible that the issues of less-than-sufficient training and inadequate statistical power were simultaneously applicable to the current findings. Even if that was the case, however, the combination of the two strategies - the use of more extensive training and a priori power analysis in Experiment 2-would still be an effective approach to addressing both of the issues.

\section{Experiment 2}

\section{Method}

Participants A new sample of 83 participants (11 men, 72 women) from the Cleveland State University community consented to participate in this experiment for partial course credit. They ranged in age from 18 to 30 years $(M=20.04)$. All participants reported normal or corrected-to-normal vision.

This number of participants was determined by a priori power analysis conducted with the $\mathrm{G}^{*}$ Power program (version 3.1; Faul, Erdfelder, Lang, \& Buchner, 2007). Assuming that the 
offset-biasing manipulations, if sufficiently effective, would produce the interaction between trial type and attentional bias condition in Block 2 that is of the same magnitude as observed in Block 1 of Experiment $1\left(\eta_{\mathrm{p}}{ }^{2}=0.172\right.$ for reaction time), Experiment 2 should have sufficient power to detect an interaction of this size with $\alpha=.05$. Given the number of betweenparticipant conditions and repeated measures in the current experiments, when reasonably high power $(1-\beta=0.80)$ is required, this analysis showed that 64 participants $(16$ per condition) would need to be tested in Experiment 2. To be conservative, we set the required power level even higher in the analysis $(1-\beta=0.90)$, which resulted in 80 participants (20 per condition) in Experiment 2.

\section{Materials, design, procedure, and data analysis} Experiment 2 was identical to Experiment 1 with the exception that the number of trials in each block (but not in the practice session) was doubled in Experiment 2. To do this, the two sets of 160 photograph pairs (one was used in Block 1 of Experiment 1, and the other was used in Block 2 of Experiment 1) were combined into a single set of 320 photograph pairs, and they were used for creating onset and offset trials in both Block 1 and Block 2 of Experiment 2. However, like in Experiment 1, some pairs were used just as offset trials in Block 1 of the skewed ratio and skewed ratio with instruction conditions. Thus, Experiment 2 used twice as many trials as in Experiment 1 for each manipulation-for example, in Block 1 of the skewed ratio condition, participants performed 72 onset and 248 offset trials, with the first and last 40 trials being offset trials. These 320 trials contained 64 dummy trials, 48 of which were offset trials. Similarly, in Block 1 of the neutral condition (which was identical to Block 2 of all conditions), participants viewed 160 onset and 160 offset trials. Of these, 32 onset and 32 offset trials constituted dummy trials.

\section{Results}

Participants were to be excluded from the analysis if their mean accuracy in the left/right judgment task for either onset or offset trials had been more than three standard deviations away from the mean of all participants for a given attentional bias condition. However, no participants reached this criterion and thus no one was excluded on the basis of accuracy. Data from three participants (one in the skewed ratio condition, one in the skewed ratio with instruction condition, and one in the instruction only condition) were not analyzed because miscommunication of instructions, simple fatigue, or technical issue during the experiment prevented task completion. After exclusion of these participants, each of the attentional bias conditions had 20 participants. Reaction time outliers were then removed if they were more than three standard deviations away from each participant's mean, separately for onsets and offsets. There was one outlier at the lower cutoff for one participant. At the upper cutoff, one to four outliers for each trial type were removed for 46 participants. Removal of these outliers did not count against participants' accuracy data. Exclusion of the outliers did not cause results to vary. Mean reaction times were then computed for each trial type and for each attentional bias condition, based on trials in which correct responses were made.

Block 1 As in Experiment 1, data from the first block were analyzed to determine the efficacy of the offset-biasing manipulations.

Reaction time Figure 5a shows mean reaction times in Block 1 as a function of trial type and attentional bias condition. Participants in the neutral and instruction only conditions detected onsets more quickly than offsets, whereas those in the skewed ratio and skewed ratio with instruction conditions exhibited the opposite pattern. In the ANOVA, the main effect of attentional bias was not significant, $F(3,76)=0.182, p=$ $0.907, \eta_{\mathrm{p}}{ }^{2}=0.007$, and the main effect of trial type was marginally significant, $F(1,76)=3.007, p=0.083, \eta_{\mathrm{p}}{ }^{2}=0.039$, suggesting that in the first block there was an overall trend in detecting onsets faster than offsets. More importantly, the interaction of trial type and attentional bias was significant, $F(3$, 76) $=9.374, p<0.001, \eta_{\mathrm{p}}{ }^{2}=0.270$. Simple effects analyses revealed significantly shorter reaction times to onset trials than to offset trials in the neutral condition, $p<0.001$, and in the instruction only condition, $p=0.004$. Significantly shorter reaction times to offset trials than to onset trials were found in the skewed ratio with instruction condition, $p=0.009$. There was no significant difference between onset and offset trials for the skewed ratio condition, $p=0.536$. Thus, although the instruction by itself might not have been effective, the other two manipulations clearly modulated onset primacy in Block 1-the dominance of offset trials in the skewed ratio condition eliminated onset primacy, and when the instruction was added to this manipulation, clear bias toward offsets was observed.

Accuracy Mean accuracy scores are plotted in Fig. 5b as a function of trial type and attentional bias condition. No clear preference for one trial type was present in the neutral and instruction only conditions, but in the skewed ratio and skewed ratio with instruction conditions, accuracy in offset trials was greater than that in onset trials. The main effect of attentional bias was not significant, $F(3,76)=1.494, p=$ $0.223, \eta_{\mathrm{p}}{ }^{2}=0.056$, but the main effect of trial type as well as the interaction between trial type and attentional bias were significant, $F(1,76)=15.059, p<0.001, \eta_{\mathrm{p}}{ }^{2}=0.165$, and $F(3$, $76)=5.942, p=0.001, \eta_{\mathrm{p}}{ }^{2}=0.190$, respectively. Simple effects analyses revealed significantly higher accuracy for 

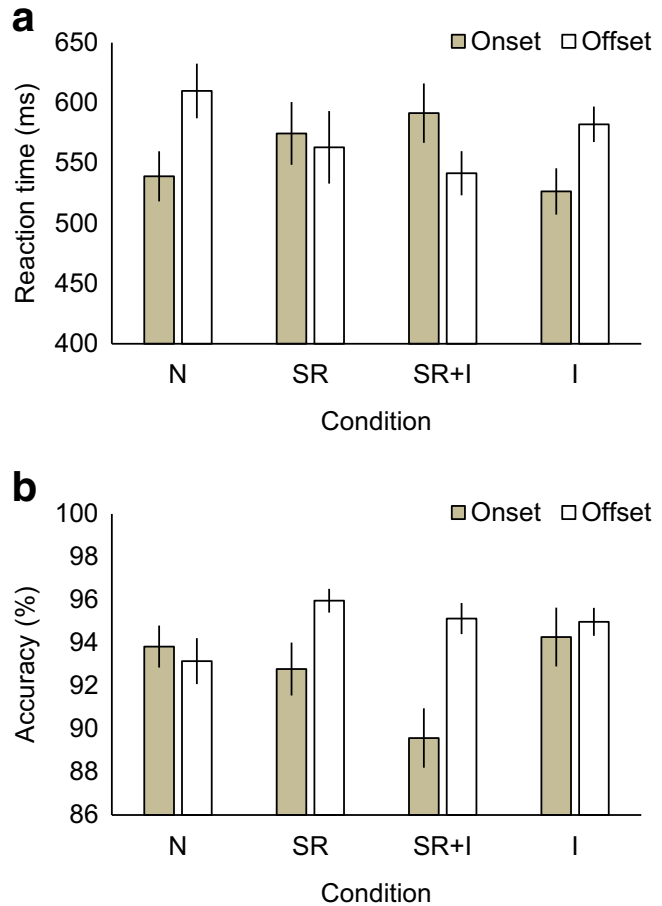

Fig. 5 Mean reaction time (a) and accuracy (b) in the first block of trials in Experiment 2 as a function of attentional bias condition and trial type. Condition labels are defined as follows: $\mathrm{N}=$ neutral condition; $\mathrm{SR}=$ skewed ratio condition; $\mathrm{SR}+\mathrm{I}=$ skewed ratio with instruction condition; and $\mathrm{I}=$ instruction only condition. Error bars represent \pm 1 standard error of the mean

offset trials compared to onset trials in the skewed ratio condition, $p=.006$, and in the skewed ratio with instruction condition, $p<0.001$. There was no significant difference in accuracy between onset and offset trials in the neutral condition, $p$ $=0.549$, and in the instruction only condition, $p=0.533$. Consistent with the reaction time data reported above, these accuracy data suggest that the instruction alone could not exert observable effects in this experiment, but the other manipulations, especially greater exposure to offset trials combined with the instruction, were effective in inducing offsetdetection bias in Block 1.

Block 2 Data from the second block were analyzed in the same manner as in Experiment 1. The data from the entire block were examined first, and then they were divided into sub-blocks to investigate whether the effects of offset-biasing manipulations changed as participants went through trials.

Reaction time Figure 6a shows mean reaction times in the second block as a function of trial type and attentional bias condition. Participants in the neutral and instruction only conditions performed onset trials more quickly than offset trials. On the other hand, those in the skewed ratio and skewed ratio with instruction conditions yielded similar reaction times to onset and offset trials. In Block 2, the main effect of attentional
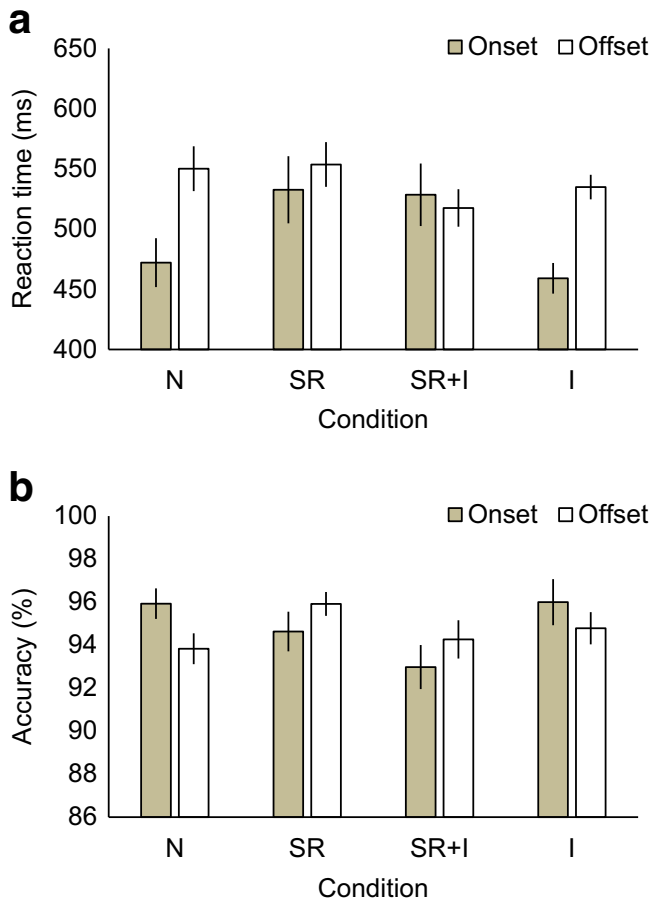

Fig. 6 Mean reaction time (a) and accuracy (b) in the second block of trials in Experiment 2 as a function of attentional bias condition and trial type. Condition labels are defined as follows: $\mathrm{N}=$ neutral condition; $\mathrm{SR}=$ skewed ratio condition; $\mathrm{SR}+\mathrm{I}=$ skewed ratio with instruction condition; and $\mathrm{I}=$ instruction only condition. Error bars represent \pm 1 standard error of the mean

bias was not significant, $F(3,76)=1.355, p=0.263, \eta_{\mathrm{p}}{ }^{2}=$ 0.051 , but both the main effect of trial type and the interaction of trial type and attentional bias were significant, $F(1,76)=$ 20.309, $p<0.001, \eta_{\mathrm{p}}{ }^{2}=0.211$, and $F(3,76)=5.730, p=$ $0.001, \eta_{\mathrm{p}}^{2}=0.184$, respectively. Simple effects analyses revealed significantly shorter reaction times to onset trials than to offset trials in the neutral and instruction only conditions, $p \mathrm{~s}$ $<0.001$. There was no significant difference between onset and offset trials for the skewed ratio condition, $p=0.252$, and for the skewed ratio with instruction condition, $p=0.548$. These results suggest that, unlike in Experiment 1, biasing manipulations did carry over from Block 1 to Block 2 in Experiment 2, wherein greater exposure to offset trials with and without an instruction to attend to offsets was able to eliminate onset primacy. However, when the instruction was used by itself, it did not override onset primacy.

Accuracy Mean accuracy scores are plotted in Fig. 6b as a function of trial type and attentional bias condition. Participants were more accurate in onset trials than in offset trials in the neutral and instruction only conditions, whereas they tended to be more accurate in offset trials than in onset trials in the skewed ratio and skewed ratio with instruction conditions. Neither the main effect of attentional bias nor the main effect of trial type were significant, $F(3,76)=1.180, p=$ $0.323, \eta_{\mathrm{p}}{ }^{2}=0.045$, and $F(1,76)=0.274, p=0.602, \eta_{\mathrm{p}}{ }^{2}=$ 
0.004 , respectively. The interaction between trial type and attentional bias was significant, $F(3,76)=6.022, p<0.001$, $\eta_{\mathrm{p}}{ }^{2}=0.192$. Simple effects analyses revealed that accuracy for onset trials was significantly higher than accuracy for offset trials in the neutral condition, $p=0.004$, and that the trend in the same direction was present in the instruction only condition, $p=0.090$. Offsets were detected with marginally higher accuracy compared to onsets in the skewed ratio condition, $p$ $=0.074$, and in the skewed ratio with instruction condition, $p$ $=0.073$. These data corroborate the findings from the reaction time results reported above, showing that onset primacy was certainly diminished in the skewed ratio and skewed ratio with instruction conditions. The accuracy data also were consistent with the reaction time data in that the instruction was not able to clearly modulate onset primacy when it was not accompanied with the increased number of offset trials.

Split-block analysis Because of the larger number of trials in Experiment 2 than in Experiment 1, the block was divided into quarters instead of splitting it in half. The same number of trials was used for creating a sub-block for the split-block analysis in both experiments (80 trials per quarter in Experiment 2 or per half in Experiment 1). As in the same analysis in Experiment 1, due to the random presentation of trial types, different numbers of onset and offset trials were used for computing mean reaction times and accuracy for each participant and for each quarter. However, it was confirmed that there was no significant variation in these numbers between participants and quarters. The mean numbers of onset and offset trials in each quarter of the block were as follows: 32.61 onset and 31.71 offset trials in the first quarter; 31.82 onset and 32.05 offset trials in the second quarter; 32.19 onset and 31.56 offset trials in the third quarter; and 31.10 onset and 32.76 offset trials in the fourth quarter.

Figure 7 shows mean reaction times and accuracy in each quarter of Block 2 as a function of trial type and attentional bias condition. As shown in Fig. 7a-d, patterns of reaction time data across trial types and conditions exhibited little variation between quarters. The same was largely the case for the accuracy data, with the notable exception in the skewed ratio with instruction condition: In this condition, accuracy for offsets was distinctly higher than that for onsets in the first quarter (Fig. 7e), but this difference disappeared as early as in the second quarter (Fig. $7 \mathrm{f}-\mathrm{h}$ ).

These data were analyzed by two mixed ANOVAs (one for reaction time and one for accuracy) with trial type and subblock (first, second, third, and fourth quarters) as withinparticipant factors and attentional bias as a betweenparticipant factor. Because the sphericity assumption was not always met in these ANOVAs, $p$ values were corrected by the Greenhouse-Geisser method when appropriate. In the reaction time data, the only effect including the sub-block variable that reached significance was the main effect of sub-block, $F(3$,
228) $=5.345, p=0.004, \eta_{\mathrm{p}}{ }^{2}=0.066$; there was an tendency that overall mean reaction times became shorter in later quarters. All other effects including the sub-block variable were not significant, $F_{\mathrm{s}}<1.382, p \mathrm{~s}>0.216, \eta_{\mathrm{p}}{ }^{2} \mathrm{~s}<0.053$, suggesting that the effects of trial type and attentional bias did not differ between quarters. The other effects that were significant in the ANOVA were the main effect of trial type, $F(1,76)=$ 18.871, $p<0.001, \eta_{\mathrm{p}}{ }^{2}=0.199$, and the interaction between trial type and attentional bias, $F(3,76)=5.915, p=0.001, \eta_{\mathrm{p}}{ }^{2}$ $=0.189$. As for the accuracy data, the three-way interaction between trial type, attentional bias, and sub-block was marginally significant, $F(9,228)=1.712, p=0.088, \eta_{\mathrm{p}}{ }^{2}=0.063$, and the two-way interaction between trial type and sub-block was significant, $F(3,228)=2.719, p=0.046, \eta_{\mathrm{p}}{ }^{2}=0.035$. These results are consistent with the observation that preference for onset and offset trials changed depending on when the trials were performed within Block 2, and this interaction differed to some extent between attentional bias conditions. More specifically, in earlier quarters in the skewed ratio and skewed ratio with instruction conditions, participants were more accurate in offset detection than in onset detection; however, the advantage of offset trials dissipated in later quarters. This pattern was most clearly seen in the skewed ratio with instruction condition. On the other hand, the patterns of results for the neutral and instruction only conditions were consistent across quarters, showing onset primacy throughout the block. In sum, these results suggest that offset-biasing effects on accuracy were carried over from Block 1 to Block 2, in particular in the skewed ratio with instruction condition, but the effects were fairly short-lived. Other significant effects in this ANOVA were the main effect of sub-block, $F(3,228)=3.032$, $p=0.036, \eta_{\mathrm{p}}{ }^{2}=0.038$ (overall accuracy slightly declined in the fourth quarter); and the interaction between trial type and attentional bias, $F(3,76)=9.351, p<0.001, \eta_{\mathrm{p}}{ }^{2}=0.270$.

\section{Discussion}

Results from the second experiment involving longer training and testing blocks provided more detailed insights into the default mode hypothesis and the attentional modulation hypothesis. With more extensive training in prioritizing offset detection, participants in Experiment 2-in particular those in the skewed ratio and skewed ratio with instruction conditions - exhibited no clear tendency of detecting one type of trials more efficiently than the other in Block 2. Given that participants in the neutral condition consistently showed onset primacy, the lack of onset primacy in the skewed ratio and skewed ratio with instruction conditions can be interpreted to mean that participants were able to adjust their attentional priority according to the specific behavioral context (i.e., they encountered more offsets than onsets in Block 1) and the 

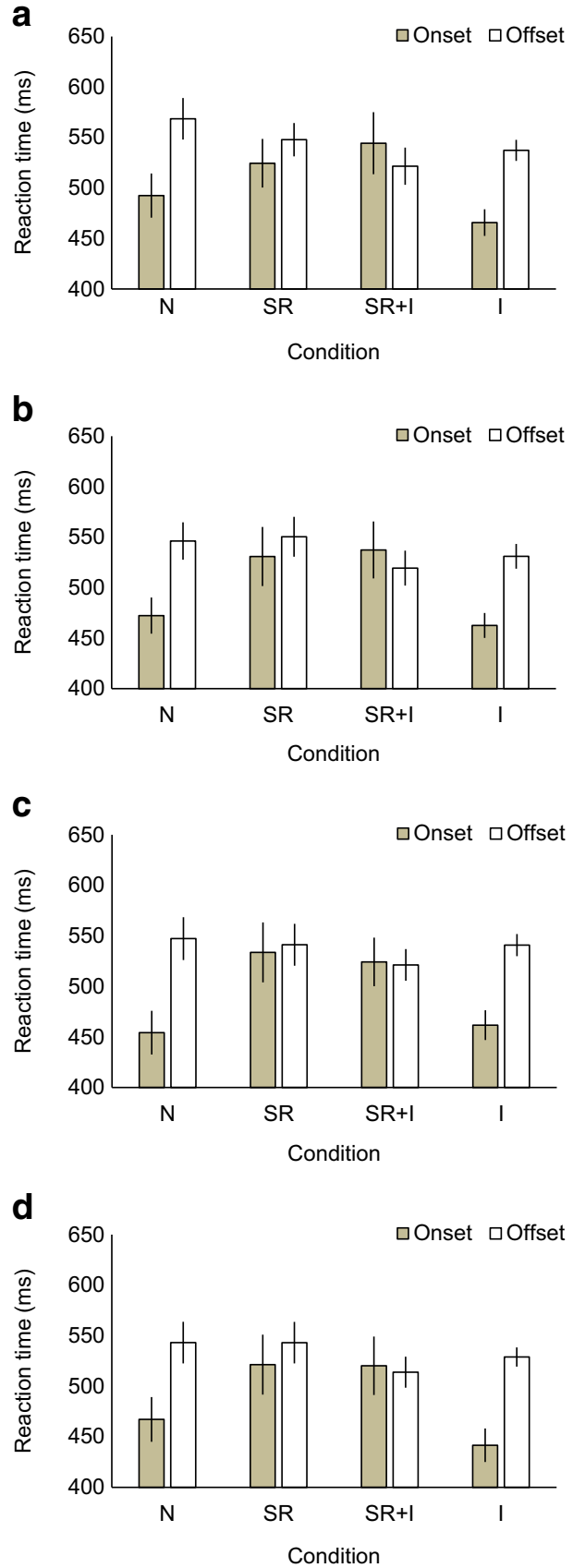

Fig. 7 Mean reaction time (a-d) and accuracy (e-h) in each quarter of the second block of trials of Experiment 2 as a function of attentional bias condition and trial type. Panels $\mathrm{A}$ and $\mathrm{E}$ show data from the first quarter, panels $\mathrm{B}$ and $\mathrm{F}$ show data from the second quarter, panels $\mathrm{C}$ and $\mathrm{G}$ show data from the third quarter, and panels $\mathrm{D}$ and $\mathrm{H}$ show data from the fourth

top-down control (i.e., the instruction to attend to offsets). These results offer stronger support for the attentional modulation hypothesis than those of Experiment 1, in which evidence for attentional flexibility was found only during the training block.

At the same time, the fact that even with the extensive training onset primacy was only diminished at best- that is, it was not reversed such that offsets were detected more
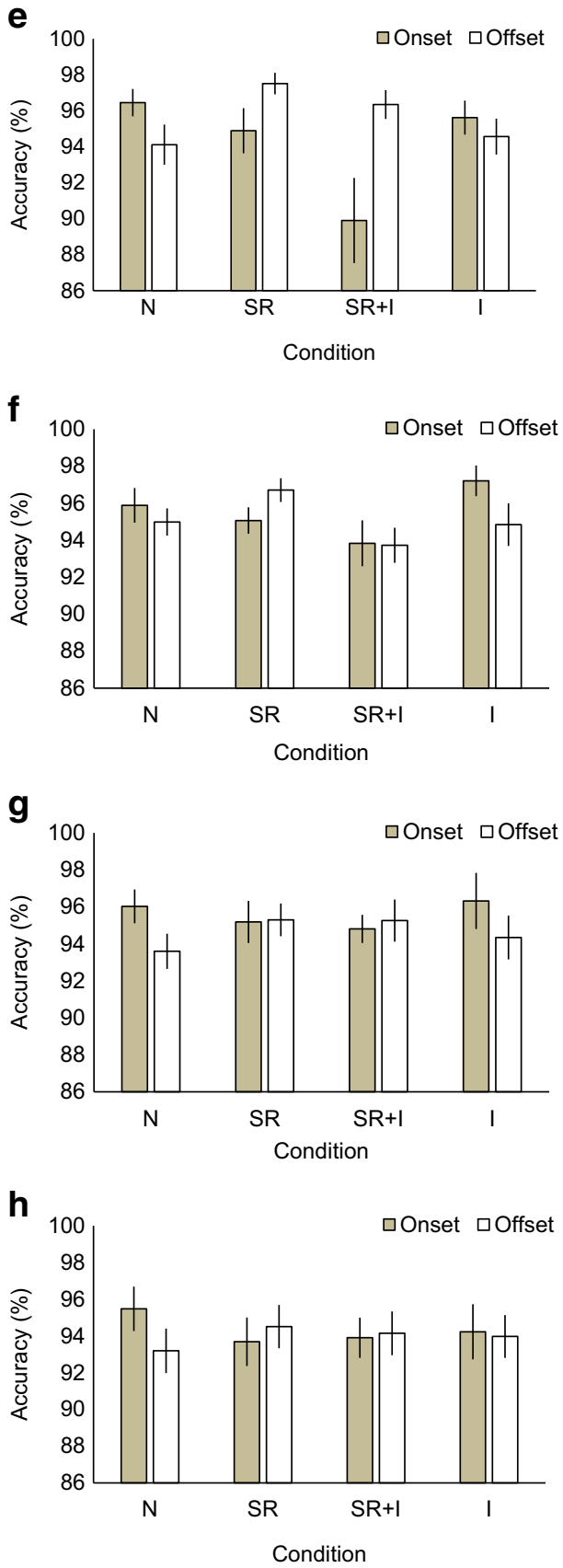

quarter. Condition labels are defined as follows: $\mathrm{N}=$ neutral condition; $\mathrm{SR}$ = skewed ratio condition; $\mathrm{SR}+\mathrm{I}=$ skewed ratio with instruction condition; and $\mathrm{I}=$ instruction only condition. Error bars represent 1 standard error of the mean

quickly and accurately than onsets - indicates how robust onset primacy is as the default mode of attention. Results of the split-block analysis corroborate this notion by showing that effects of offset-prioritizing manipulations were more clearly seen in early trials of Block 2, but they started disappearing within the block as participants performed more trials. This temporal pattern is consistent with the view that participants were going back to normal (i.e., the default onset-prioritizing 
mode) as they became aware that the environment stopped providing an incentive to pay greater attention to offsets (i.e., in Block 2, offset trials were no longer dominant). Importantly, in contrast to offsets, onsets were detected with greater speed and accuracy even when they were no more common than offsets in the neutral condition. This suggests that the primacy of onset in visual change detection is not a simple consequence of adapting to specific behavioral contexts; rather, it does suggest that onset primacy is more intrinsically built into the attentional system as one of its essential properties.

In Experiment 2, the instruction to attend to offsets had little effect, even in Block 1. Considering that this instruction was given just once at the beginning of the experiment, longer blocks of Experiment 2 might have caused greater decrement of the effect of the instruction. In the instruction only condition, participants were exposed to the same number of onset and offset trials in both blocks, and thus they might have perceived little relevance of the instruction. However, when the utility of the instruction was instantiated by frequent encounter with offset trials, this made the most effective offsetbiasing condition in this study (i.e., the skewed ratio with instruction condition, especially in Block 1). The effect of the instruction and that of the skewed ratio manipulation were not simply additive, suggesting the potential importance of interaction between top-down factors (e.g., instructions, behavioral goals, and internal motivation) and bottom-up cues (e.g., certain environmental situations that make detection of non-onset events advantageous) in modulating observers' attentional priority.

\section{General Discussion}

The present study was designed to gain insights into why object onset often is detected more efficiently than object offset in visual scenes. Specifically, we contrasted two hypotheses. The default mode hypothesis posited that the natural selection shaped the human attentional system that pays increased attention to object onsets in most situations. On the other hand, the attentional modulation hypothesis postulated that observers give attentional priority to any type of visual event that is most relevant to their current environment. To test these hypotheses, we had participants detect object onsets and offsets under conditions that induced bias toward prioritizing detection of offsets. Results showed that experimental manipulations produced offset-detection bias to some extent, especially when they were implemented forcefully by combining multiple types of manipulations (i.e., the skewed ratio with instruction condition) and by giving participants extensive training in prioritizing offset detection (Experiment 2). However, even under the best condition, the offset-biasing effects started fading away soon after the manipulations (in particular the presentation of a disproportionally large number of offset trials) were ceased, and performance in onset trials became increasingly better relative to performance in offset trials later in the experiments. These results suggest that increased attention to offsets occurred only as a temporary adaptation to specific behavioral contexts, and participants' attentional mode reverted back to the baseline (i.e., prioritization of onset detection) when the biased contexts were no longer present. These findings lend support for the default mode hypothesis, suggesting that the human attentional system is tuned to detect object onsets under normal circumstances, and overwriting this default setting might require continuous exposure to conditions under which detection of nononset events is optimal. At the same time, the present findings did not disqualify the attentional modulation hypothesis because this study showed that greater-than-usual attention can be allocated to object offsets at least transiently.

The robustness of onset primacy demonstrated in the present study suggests that it might not be appropriate to conceptualize object offset as a simple reverse of object onset; instead, it is possible that onset is represented as a fundamentally distinct form of visual events in the human attentional system. As discussed in the introduction, this can be understood from an evolutionary point of view such that without attending to an onset, observers cannot know if it is of threatening or harmless nature. If an onset poses a threat, they must react quickly to increase chances of survival (Cole et al., 2003). On the other hand, given that an offset is the deletion of something previously viewed, its occurrence may not command attention so immediately because its exit does not necessarily impose a danger.

This discussion is relevant to the debate about what makes abrupt onsets such prominent search targets in a visual array. Results from visual search studies suggest that a new object itself, not sensory transients associated with its appearance, captures attention (Jonides \& Yantis, 1988; Yantis \& Jonides, 1984). This claim is based on findings that observers tend to detect newly appearing stimuli more quickly or accurately than re-appearing old stimuli even when these stimuli are equated as to low-level sensory characteristics as much as possible (Davoli et al., 2007; Yantis \& Hillstrom, 1994). However, this view has been challenged by the finding that new objects presented without local luminance change do not always capture attention (Franconeri et al., 2005; Hollingworth et al., 2010). As such, it has been difficult to reconcile these opposing views by using results from visual search studies; perhaps a better approach may be to assume the roles of both sensory transients and new objects themselves and focus on the way they interact to capture attention (Atchley, Kramer, \& Hillstrom, 2000). Investigation of onset primacy in the context of change blindness may provide a new perspective to this debate. In change blindness paradigms, the role of sensory transients is generally minimized because they 
are not distinctively localized to a specific object. For example, in a flicker paradigm, the entire scene abruptly appears after an intervening blank screen. The fact that onset primacy was clearly observed in the present one-shot flicker paradigm suggests that the robust detection of onsets is attributed, at least to some extent, to the presence of new objects per se. By further integrating the visual search literature with the change blindness literature, contributions of new objects and sensory transients to onset primacy would be more precisely determined.

The attentional system is a product of the interplay between top-down control and bottom-up information processing (Yantis, 2005). Using top-down control to guide cognition and behavior, individuals are able to volitionally interact with an environment. However, if an event should occur such that they need to react to it accordingly, attentional resources are quickly deployed from their top-down behavioral goals to the salient event, when informed by stimulus-driven processes in the perceptual systems. The hypotheses tested in the present study did not necessarily stem from theoretical differences in how top-down and bottom-up processes guide attention, but they do offer insight into these different processing mechanisms. For example, the present results showed that instructions to prioritize offset detection did not override the default mode of attention (in which onset detection is prioritized), especially when the instructions constituted the sole attention-modulating factor. This finding demonstrates a case in which top-down signals fall short of taking control of attention. This is consonant with the view that bottom-up cues are generally more effective in capturing observers' attention than top-down cues (Theeuwes, 2004).

It should be noted, however, that the literature also provides evidence that top-down signals can win a competition against bottom-up signals in controlling attention. For example, Leber and Egeth (2006b) challenged Theeuwes' (2004) position by demonstrating that participants who were provided with an attentional search set successfully avoided interference from a salient distractor. This finding highlights dominance of topdown control over bottom-up attentional capture. Additionally, top-down resources can control selective attention such that observers attend to the aspects of a display that are most likely to elicit an external reward (Shomstein \& Johnson, 2013). In this manner, participants' attention can be guided by reward or motivational mechanisms. In the present study, the purely top-down manipulation (i.e., instruction alone) was not effective for altering the default onsetprioritizing mode of attention, but when it was combined with a bottom-up cue (i.e., offset being the dominant type of change in an environment), the instruction made additional impact on participants' attentional priority over and above the effect of the bottom-up cue. Taken together, although participants in the present study were not able to overcome onset primacy even with the explicit instruction to attend to offsets, this should not be taken as showing general weakness of topdown signals in modulating human attention. Rather, the present results suggest that motivational influences would need to be sufficiently strong and ideally reinforced with environmental demands to encourage a switch to an alternative mode of attentional processing.

Finally, it should be pointed out that the present study utilized relatively brief training for better attending to offsets. Even in Experiment 2, participants performed only 320 trials in Block 1. Although this amount of trials was sufficient for eliminating onset primacy in some conditions, the question remains as to what would happen when observers are trained much more extensively for overriding the default onsetprioritizing mode. Studies asking this question may be able to draw from real-world examples, assuming that the ability to attend to a certain type of visual events (e.g., offsets) is general enough and the identity of disappearing objects is less critical. For instance, professional lifeguards have both an internal (topdown) motivation to be vigilant to offsets (drowning swimmers) and an environmental (bottom-up) context that calls for quick detection of the offsets. This situation could create an impressively long training phase that has the properties required for making the training effective (i.e., co-occurrence of behavioral goals and environmental demands). By examining the degree of attentional flexibility in individuals who routinely pay attention to non-onset events for their profession or for certain intrinsic purposes, future research could examine the true potential of the human attentional system in flexibly allocating attention to various types of visual events.

Acknowledgments The authors thank Eric Allard, Conor $\mathrm{M}^{\mathrm{c} L e n n a n,}$ Ernest Park, and Andrew Slifkin for their helpful comments and feedback, and Istvan Karacson, Tom Kruse, Abigail Landreth, and Kaitlyn Lewanski for assistance with participant recruitment and data collection.

\section{References}

Atchley, P., Kramer, A. F., \& Hillstrom, A. P. (2000). Contingent capture for onsets and offsets: Attentional set for perceptual transients. Journal of Experimental Psychology: Human Perception and Performance, 26, 594-606. doi:10.1037/0096-1523.26.2.594

Bugg, J. M., Jacoby, L. L., \& Toth, J. P. (2008). Multiple levels of control in the Stroop task. Memory \& Cognition, 36, 1484-1494. doi:10.3758/MC.36.8.1484

Chua, F. K. (2013). Attentional capture by onsets and offsets. Visual Cognition, 21, 569-598. doi:10.1080/13506285.2013.812700

Chun, M. M., \& Jiang, Y. (1998). Contextual cueing: Implicit learning and memory of visual context guides spatial attention. Cognitive Psychology, 36, 28-71. doi:10.1006/cogp.1998.0681

Cole, G. G., Kentridge, R. W., Gellatly, A. R. H., \& Heywood, C. A. (2003). Detectability of onsets versus offsets in the change detection paradigm. Journal of Vision, 3, 22-31. doi:10.1167/3.1.3

Cole, G. G., Kentridge, R. W., \& Heywood, C. A. (2004). Visual salience in the change detection paradigm: The special role of object onset. Journal of Experimental Psychology: Human Perception and Performance, 30, 464-477. doi:10.1037/0096-1523.30.3.464 
Cole, G. G., \& Kuhn, G. (2010). Attentional capture by object appearance and disappearance. The Quarterly Journal of Experimental Psychology, 63, 147-159. doi:10.1080/17470210902853522

Cole, G. G., Liversedge, G., \& Simon, P. (2006). Change blindness and the primacy of object appearance. Psychonomic Bulletin \& Review, 13, 588-593. doi:10.3758/BF03193967

Davoli, C. C., Suszko, J. W., \& Abrams, R. A. (2007). New objects can capture attention without a unique luminance transient. Psychonomic Bulletin \& Review, 14, 338-343. doi:10.3758/BF03194074

Donaldson, M. J., \& Yamamoto, N. (2012). Detection of object onset and offset in naturalistic scenes. In C. Stachniss, K. Schill, \& D. Uttal (Eds.), Lecture Notes in Computer Science: Vol. 7463. Spatial Cognition VIII (pp. 451-460). Berlin, Germany: Springer-Verlag. doi:10.1007/978-3-642-32732-2_29

Faul, F., Erdfelder, E., Lang, A.-G., \& Buchner, A. (2007). G*Power 3: A flexible statistical power analysis program for the social, behavioral, and biomedical sciences. Behavior Research Methods, 39, 175-191. doi:10.3758/BF03193146

Folk, C. L., Remington, R. W., \& Johnston, J. C. (1992). Involuntary covert orienting is contingent on attentional control settings. Journal of Experimental Psychology: Human Perception and Performance, 18(1030), 1044. doi:10.1037/0096-1523.18.4.1030

Franconeri, S. L., Hollingworth, A., \& Simons, D. J. (2005). Do new objects capture attention? Psychological Science, 16, 275-281. doi:10.1111/j.0956-7976.2005.01528.x

Hollingworth, A., Simons, D. J., \& Franconeri, S. L. (2010). New objects do not capture attention without a sensory transient. Attention, Perception, \& Psychophysics, 72, 1298-1310. doi:10.3758 /APP.72.5.1298

Jonides, J., \& Yantis, S. (1988). Uniqueness of abrupt visual onset in capturing attention. Perception \& Psychophysics, 43, 346-354. doi:10.3758/BF03208805

Kelley, T. A., \& Yantis, S. (2009). Learning to attend: Effects of practice on information selection. Journal of Vision, 9(7), 16. doi:10.1167/9.7.16
Leber, A. B., \& Egeth, H. E. (2006a). Attention on autopilot: Past experience and attentional set. Visual Cognition, 14, 565-582. doi:10.1080/13506280500193438

Leber, A. B., \& Egeth, H. E. (2006b). It's under control: Top-down search strategies can override attentional capture. Psychonomic Bulletin \& Review, 13, 132-128. doi:10.3758/BF03193824

Levin, D. T., \& Varakin, D. A. (2004). No pause for a brief disruption: Failure of visual awareness during ongoing events. Consciousness and Cognition: An International Journal, 13, 363-372. doi:10.1016 /j.concog.2003.12.001

Shomstein, S., \& Johnson, J. (2013). Shaping attention with reward: Effects of reward on space-and object-based selection. Psychological Science, 24, 2369-2378. doi:10.1177/0956797613490743

Simons, D. J., \& Rensink, R. A. (2005). Change blindness: Past, present, and future. Trends in Cognitive Sciences, 9, 16-20. doi:10.1016/j. tics.2004.11.006

Theeuwes, J. (2004). Top-down search strategies cannot override attentional capture. Psychonomic Bulletin and Review, 11, 65-70. doi:10.3758/BF03206462

Yantis, S. (2005). How visual salience wins the battle for awareness. Nature Neuroscience, 8, 975-977. doi:10.1038/nn0805-975

Yantis, S., \& Hillstrom, A. P. (1994). Stimulus-driven attentional capture: Evidence from equiluminant visual objects. Journal of Experimental Psychology: Human Perception and Performance, 20, 95-107. doi:10.1037/0096-1523.20.1.95

Yantis, S., \& Jonides, J. (1984). Abrupt visual onsets and selective attention: Evidence from visual search. Journal of Experimental Psychology: Human Perception and Performance, 10, 601-621. doi:10.1037/0096-1523.16.1.121

Yantis, S., \& Jonides, J. (1990). Abrupt visual onsets and selective attention: Voluntary versus automatic allocation. Journal of Experimental Psychology: Human Perception and Performance, 16, 121-134. doi:10.1037/0096-1523.16.1.121 